\title{
HIGH BCR-ABL1 GENE PERCENTAGE AT TIME OF PRESENTATION: A TOOL TO PREDICT FAILURE IN ACHIEVING EARLY MOLECULAR RESPONSE IN CHRONIC MYELOID LEUKEMIA (CML): A TERTIARY CARE CENTER EXPERIENCE
}

\author{
Amjad Khan, Riaz Ahmed, Sarah Fatimah*, Muhammad Nadeem, Shama Iqbal**, Sayed Tanveer Abbas Gilani***, \\ Huma Amjad****
}

Combined Military Hospital/National University of Medical Sciences (NUMS) Rawalpindi Pakistan, *Armed Forces Institute of Pathology/ National University of Medical Sciences (NUMS) Rawalpindi Pakistan, ${ }^{* *}$ Naserullah Baber Memorial Hospital, Peshawar Pakistan, ${ }^{* * *}$ Armed Forces Institute of Cardiology/ National Institute of Heart Disease (AFIC/NIHD) National University of Medical Sciences (NUMS) Rawalpindi Pakistan, ****Basis Health Unit, Swabi Pakistan

\begin{abstract}
Objective: To determine the relationship of baseline quantitative BCR ABL1 gene percentage and therapeutic response i.e. Early Molecular Response (EMR) at 3 months with first generation Tyrosine kinase inhibitors (Imatinib) in patients with Chronic Myeloid Leukemia (CML) in chronic phase (CP)

Study Design: Prospective observational study.

Place and Duration of Study: Combined Military Hospital, Rawalpindi, Pakistan, and Armed Forces Institute of Pathology Rawalpindi, Pakistan from Oct 2017 to Oct 2019.

Methodology: One hundred and seventy patients, 18 years of age or older with newly diagnosed Chronic Myeloid Leukemia (CML) in chronic phase (CP) with quantitative baseline BCR-ABL (IS) transcript were included in the study. All enrolled patients were placed on Imatinib therapy ( $400 \mathrm{mg} /$ day) and Reverse transcription polymerase chain reaction (RT-PCR) for BCR ABL transcript was repeated at 3 months to document EMR (BCR-ABL (IS) $<10 \%$ ). Patients who were in accelerated/blast phase, or already taking any Tyrosine Kinase Inhibitors (TKI) or chemotherapy were excluded from the study

Results: In our study 101 (59.4\%) patients achieved early molecular response. Out of these 80 (70.8\%) patients with BCR-ABL $<50 \%$ at baseline value showed early molecular response. However, only 21 (36.8\%) with BCR$\mathrm{ABL}>50 \%$ at baseline achieved early molecular response $(p$-value $<0.001)$.

Conclusion: A significant number of patients achieved early molecular response with Imatinib therapy that had BCR ABL below $50 \%$, however those with baseline BCR ABL $>50 \%$, the rate of EMR was comparatively lower.
\end{abstract}

Keywords: Chronic Myeloid Leukemia, Early molecular response, Imatinib.

\footnotetext{
This is an Open Access article distributed under the terms of the Creative Commons Attribution License (http://creativecommons.org/licenses/by/4.0), which permits unrestricted use, distribution, and reproduction in any medium, provided the original work is properly cited.
}

\section{INTRODUCTION}

Chronic myeloid leukemia (CML) is a myeloproliferative neoplasm that has translocation between chromosome 22 and chromosome 91. CML accounts for about $15-20 \%$ of adult leukaemias with a slight male predominance. About $85 \%$ of patients present in chronic phase and if left untreated the disease usually progresses to accelerated or blast phase in three to five years, which is associated with unfavourable prognosis and poor clinical outcomes ${ }^{2}$.

CML usually presents with constitutional symptoms (i.e. lowgrade fever, weight loss and

Correspondence: Dr Amjad Khan, Classified Medical Specialist, Department of Oncology, CMH Rawalpindi Pakistan

Received: 20 Feb 2020; revised received: 10 Apr 2020; accepted: 22 Apr 2020 night sweats), splenomegaly and a high WBC count. Characteristic pathogenesis of the disease is $t(9 ; 22)$ which results in production of Philadelphia chromosome which is detected by RT-PCR and is present in approximately $90-95 \%$ of CML patients. Approximately 5\% patients have atypical transcript of BCR ABL which is detected by FISH (fluorescence in situ hybridization) ${ }^{3}$.

Sensitive residues of tyrosine are carried close to adjacent kinase domains of ABL1, which contribute to autophosphorylation and activation. These changes result in constitutive activation of ABL1 resulting in loss of growth inhibition, decreased apoptosis and decreased adherence to stromal cells in the bone marrow ${ }^{4}$. In CML-CP, Imatinib is given as first line treatment in most 
patients however, in Advance Phase (AP) second generation TKI like Nilotinib or Dasatinib are preferred $^{5}$. Response to treatment is assessed at 3 months as per ELN 2013 guideline and NCCN guidelines ${ }^{6,7}$.

The treatment target has recently been switched to early molecular reaction (EMR), i.e. BCR-ABL1 transcript $\leq 10 \%$ at 3 months as a deeper response and clinical improvement forecast ${ }^{8}$. After 3 months of imatinib therapy EMR was stated by a study as a $77.6 \%(n=410 / 528)^{8}$. Usually EUTOS Score is calculated to determine probability of achieving Complete cytogenetic response at 18 months 9 . We planned this study to determine the response of TKI therapy in association with baseline BCL ABL percentage with EMR in our study population.

\section{METHODOLOGY}

This was a prospective observational study done at the department of medical Oncology, Combined Military Hospital and Armed Forces Institute of Pathology, Rawalpindi from October 2017 to October 2019. Consecutively patients of CML in the chronic phase were recruited after the informed consent through universal sampling method. Newly diagnosed cases of CML with age $\geq 18$ years, in $\mathrm{CP}$ with quantitative $\mathrm{BCR}-\mathrm{ABL}$ were included in study. Patients in accelerated, blast phase, with atypical BCR-ABL transcript and base line $\mathrm{BCR}-\mathrm{ABL}<10 \%$ were excluded from the study. A total of 170 patients were included in the study, which was carried out after taking approval from institutional ethics review board (IERB No. 53/2017/CMH). All chosen patients had a detailed history and physical exam. For the entire baseline blood picture, blood samples were drawn with chemistry profile and quantitative RTPCR for BCR-ABL1 transcript. The sample was analyzed on sysmex $\mathrm{K} \times 21$ and BCR-ABL was done by gene expert. The international baseline BCR-ABL value was reported through by RTPCR.

In our early molecular response research, $\mathrm{BCR}-\mathrm{ABL}$ rates $<10$ percent on the IS were described three months after imatinib therapy begun.
Imatinib treatment (400 mg/day) was performed on all the patients enrolled, and after three months, quantitative BCR-ABL transcript RT-PCR was replicated according to NCCN guidelines.

All the data were analyzed using SPSS-22. Quantitative variables were measured as mean \pm SD while frequencies and percentages were calculated for qualitative variables. Paired sample t-test was applied between pretreatment and post treatment BCR-ABL levels with $p$-value $<0.05$ taken as significant. Chi-square test was applied for gender, Age groups and EMR between the two groups with BCR-ABL transcript of more than $50 \%$ and those less than $50 \%$ with $p$-value $<0.05$ taken as significant.

\section{RESULTS}

In our targeted population, there were 45 $(26.5 \%)$ females while $125(73.5 \%)$ males. The mean age of presentation was $53.3 \pm 8.0$ years (ranging from 18 to 86 years) and 116 (68.3\%) were from rural background or currently living in villages.

Out of 170 patients included in our study $113(66.5 \%)$ of patients had BCR-ABL below 50\% at time of presentation while $57(33.5 \%)$ had BCR-ABL percentage above $50 \%$. Fig- 1 showed

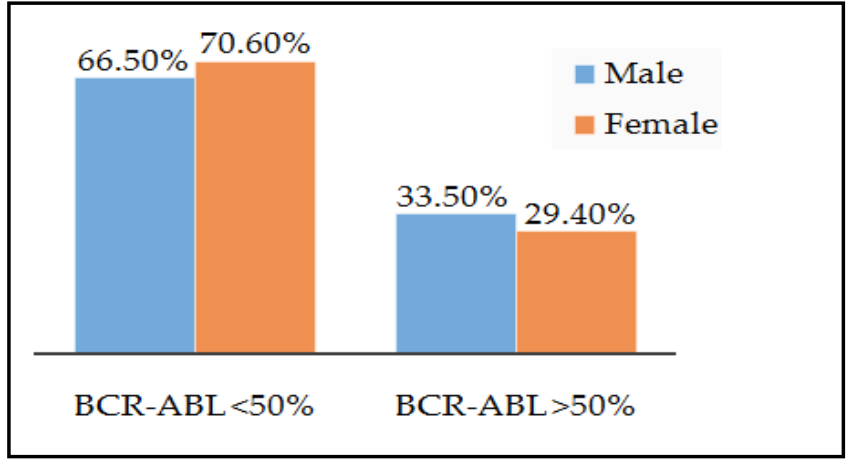

Figure-1: BCR ABL1 gene percentage at presentation.

the comparison of pretreatment BCR-ABL levels of $41 \pm 21$ IS with post treatment BCR-ABL levels of $18 \pm 19$ IS showed significant reduction after 3 months of imatinib therapy $(p<0.001)$. Overall 101 (59.4\%) patients achieved EMR while 69 (40.6\%) cases did not achieve this milestone (fig-2).

In our study population $80(70.8 \%)$ patients with BCR-ABL $<50 \%$ baseline value achieved 
EMR, however only 21 (36.8\%) with BCR-ABL $>50 \%$ at baseline showed EMR at 3 months. In total $69(40.6 \%)$ cases did not show EMR status,

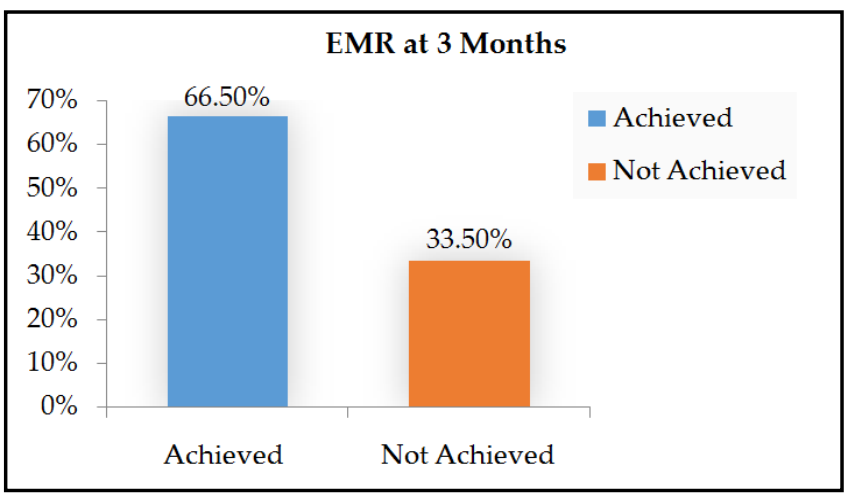

Figure-2: Early molecular response achievement at 3 months $(n=170)$.

out of which $33(29.2 \%)$ had baseline BCR-ABL $<50 \%$ while a significant proportion of $36(63.2 \%)$ having $\mathrm{BCR}-\mathrm{ABL}>50 \%$ at baseline did not achieve EMR (table).

Table: Comparison between the two groups with BCRABL transcript of $<50 \%$ and those $>50 \%(n=170)$.

\begin{tabular}{|c|c|c|c|}
\hline Parameter & $\begin{array}{c}\text { BCR-ABL } \\
<50 \% \\
(n=113) \\
n(\%)\end{array}$ & $\begin{array}{c}\text { BCR-ABL } \\
>50 \% \\
(n=57) \\
n(\%)\end{array}$ & $\begin{array}{c}p- \\
\text { value }\end{array}$ \\
\hline \multicolumn{4}{|l|}{ Gender } \\
\hline $\begin{array}{l}\text { Male } \\
\text { Females }\end{array}$ & $\begin{array}{l}84(74.3) \\
29(25.7)\end{array}$ & $\begin{array}{l}41(71.9) \\
16(28.1)\end{array}$ & 0.738 \\
\hline \multicolumn{4}{|l|}{ Age Groups } \\
\hline $\begin{array}{l}<30 \text { years } \\
30-50 \text { years } \\
>50 \text { years }\end{array}$ & $\begin{array}{c}8(7.1) \\
37(32.7) \\
68(60.2) \\
\end{array}$ & $\begin{array}{c}4(7) \\
18(31.6) \\
35(61.4)\end{array}$ & 0.886 \\
\hline \multicolumn{4}{|c|}{ Early Molecular Response } \\
\hline $\begin{array}{l}\text { Not Achieved } \\
\text { Achieved }\end{array}$ & $\begin{array}{l}33(29.2) \\
80(70.8)\end{array}$ & $\begin{array}{l}36(63.2) \\
21(36.8)\end{array}$ & $<0.001$ \\
\hline
\end{tabular}

\section{DISCUSSION}

Chronic myeloid leukemia is a disorder characterized by dysregulated production and uncontrolled proliferation of mature granulocytes. The deregulated tyrosine kinase activity in this disease has become a primary target for the treatment of this disorder and has changed the perspective of the disease entirely over the past several years since the introduction of targeted therapy (tyrosine kinase inhibitors with first generation Imatinib in 2003) ${ }^{10}$. After landmark success of imatinib, second generation Nilotinib, Bosutinib, Dasatinib and third generation drugs such as Ponatinib were developed. These agents are able to achieve long-term control of the disease in the majority of patients and have tremendously improved survival11. Despite the encouraging outcomes, In some chronic CML patients the optimal response is not achieved, as described in the current guidelines. Imatinib (IM) is generally used as first line treatment in our set up for most patients with CML in chronic phase. A part from poor compliance, drug-drug interaction, emergence of drug resistance, certain disease related factors contribute to suboptimal response ${ }^{12}$. Disease related factors like WBC, plateletts, basophils count, splenic size and the assessment of the disease risk of Sokal and Hasford scores remained clinically relevant as the required cytogenetic and molecular responses were less likely to be obtained from patients identified as high risk ${ }^{13,14}$. Early determination of patients likely to achieve early molecular response is very important, as non-responders can be shifted to second generation TKI or alternative therapies like allogenic stem cell transplant. It is well established now that early molecular response (BCR-ABL1 transcript $<10 \%$ ) at 3 months not only translates into deeper molecular responses but also predicts overall survival and progression free survival (PFS) ${ }^{15}$. Marin et al recently underlined the most important factor in detection of patients with elevated risk of disease progression to determining 3 month BCR-ABL1 transcripts ${ }^{16}$. The patients who were not able to achieve early molecular response at three months were lesslikely to achieve molecular and cytogenetic targets at 6 and 12 months, leading to decreased overall survival and progression free survival ${ }^{17}$.

In our analysis we assessed a molecular parameter easily detectable that identify CML patients less likely to achieve recommended molecular targets with Imatinib therapy. We describe the correlation between BCR-ABL1 transcript levels measured at diagnosis and the response to imatinib at three months.In our study, the higher $\mathrm{BCR}-\mathrm{ABL}$ level has been related to unsatisfactory 
drug responses, indicating lower probability of optimal answers to standard dose imatinib (400 $\mathrm{mg} /$ day). Out of 170 patients included in our study $66.5 \%$ of patients had BCR-ABL1 $<50 \%$ at time of presentation while $33.5 \%$ had BCR-ABL percentage $>50 \%$. All patients were started on Imatinib $400 \mathrm{mg} /$ day. The patients were reassessed at 3 months with quantitative BCR-ABL1 level for EMR. In our study 59.4\% patients achieved EMR while $40.6 \%$ did not achieve this important milestone. Further analysis revealed that $70.8 \%$ patients with BCR-ABL $<50 \%$ baseline value showed EMR, while only $36.8 \%$ with BCRABL1 $>50 \%$ at baseline showed early molecular response. In our study population $40.6 \%$ did not show EMR, out of these $29.2 \%$ had baseline BCRABL $<50 \%$ while a significant proportion of $63.2 \%$ had BCR-ABL $>50 \%$. Our findings are almost similar to a recently published Paolo et al study, which also found that patients with higher levels of BCR-ABL transcripts are less likely to benefit from standard IM18 dose ${ }^{18}$. In this landmark study, there was also no correlation of diagnostic and patient age transcripts of BCR-ABL, sex, hemoglobin level, social risk ratings, ph-positive metaphase and white blood cell counts. Interestingly, no link was observed between WBC diagnostic counts and BCR-ABL, which indicates the high $B C R-A B L$ values are independent of the leukemia (i.e., the amount of $\mathrm{Ph}+$ diagnostic cells).

Although there may be myriad other reasons for inferior therapeutic responses in our patient population including poor compliance, drug interactions, toxicities and genetic mutations causing TKI resistance ${ }^{19}$. Marin et al discussed various factors affecting adherence in chronic myeloid leukemia patients, including lower age group, adverse effects of TKI therapy, psychological differences among patients and perception about disease ${ }^{20}$. In our study comparison between the two groups with BCR-ABL transcript of more than $50 \%$ and those less than $50 \%$ showed no significant difference for gender and age groups but subset analysis revealed statistically significant difference for EMR showing better response for less than $50 \%$ BCR-ABL while poor response for
BCR-ABL more than $50 \%$. We report that CML patients who are less likely to benefit from IM can be determined by High BCR-ABL transcripts at diagnosis measured by RT-PCR. These patients may be considered for treatment with second generation TKIs or alternative forms of treatment at the outset as per recommended guidelines.

Our study was unique because it was carried out in Pakistani/South Asian population (which is known to have a different epidemiology with regard to $\mathrm{CML}^{21,22}$ ) and discussed association of BCR-ABL at time of presentation with EMR status. Our study was limited by the small sample size and predominantly male population. Another limitation was that we put all patients on imatinib irrespective of risk stratification due to limited availability and affordability issues in procurement of second generation TKI for all patients in upfront setting.

\section{RECOMMENDATION}

CML patients who are less likely to benefit from IM which can be determined by high BCRABL transcripts at diagnosis measured by RTPCR. These patients may be considered for alternative forms of treatment including second or third generation TKI therapy or stem cell transplant. Extensive multi-center study with high number of study population with equal representation of female and males is required for further research on subject.

\section{CONCLUSION}

EMR was achieved in more than half of patients with CML in chronic phase, being treated with Imatinib at 3 months. Although majority of patients achieved EMR with Imatinib therapy that had BCR ABL below 50\%, however those with baseline BCR ABL above $50 \%$, the rate of EMR was significantly lower.

\section{CONFLICT OF INTEREST}

This study has no conflict of interest to be declared by any author.

\section{REFERENCES}

1. Granatowicz A, Piatek CI, Moschiano E, El-Hemaidi I, Armitage JD, Akhtari M. An overview and update of chronic myeloid leu- 
kemia for primary care physicians. Korean J Fam Med 2015; 36(5): 197-202.

2. Siegel RL Miller KD, Jemal A. Cancer statistics, 2017. CA Cancer J Clin 2017; 67(1): 7-30.

3. Faderl S, Talpaz M, Estrov Z, O'Brien S, Kurzrock R, Kantarjian HM. The biology of chronic myeloid leukemia. N Engl J Med 1999; 341(3): 164

4. O'Brien S, Radich JP, Abboud CN, Akhtari M, Altman JK, Berman E, et al. Chronic myelogenous leukemia, version 1. 2015. J Natl Compr Canc 2014; 12(11): 1590-610.

5. Jain P, Kantarjian H, Cortes J. Chronic myeloid leukemia: overview of new agents and comparative analysis. Curr Treat Option 2013; 14(2): 127-43.

6. NCCN Guidelines Version 2.2020 Chronic Myeloid Leukemia, National Comprehensive Cancer Network, 2020.

7. Baccarani M, Feininger MW, Rosti G, Hochhaus A, Soverini S, Apperley JF, et al. European Leukemia Net recommendations for the management of chronic myeloid Leukemia: 2013. Blood 2013; 122(6): 872-84.

8. Hughes TP, Saglio G, Kantarjian HM, Guilhot F, Niederwieser D, Rosti G, et al. Early molecular response predicts outcomes in patients with chronic myeloid leukemia in chronic phase treated with frontline nilotinib or imatinib. Blood 2014; 123(9): 1353-60.

9. Kuntegowdanahalli LC, Kanakasetty GB, Thanky AH, Dasappa L, Jacob LA, Mallekavu SB, et al. Prognostic and predictive implications of Sokal, Euro and Euto S scores in chronic myeloid leukaemia in the imatinib era experience from a tertiary oncology centre in Southern India. E Cancer Med Sci 2016; 10(eCollection): 679-87.

10. O'Brien SG, Guilhot F, Larson RA, Gathmann I, Baccarani M, Cervantes $\mathrm{F}$, et al. Imatinib compared with interferon and lowdose cytarabine for newly diagnosed chronic-phase chronic myeloid leukemia. N Engl J Med 2003; 348(11): 994-99.

11. Jain P, Kantarjian H, Cortes J. Chronic myeloid leukemia: overview of new agents and comparative analysis. Curr Treat Option 2013; 14(2): 127-43

12. Druker BJ, Guilhot F, O'Brien SG, Gathmann I, Kantarjian H, Gattermann N, et al. Five-year follow-up of patients receiving imatinib for chronic myeloid leukemia. N Engl J Med 2006; 355(23): 2408-17.

13. Wajid AA, Zeeshan M, Mehmood F, Sharif I, Umair M, Ali A.
Early molecular response with imatinib therapy in chronic myeloid leukemia and its association with baseline while blood cell count and spleen size. Pak Armed Forces Med J 2018; 68(5): 1199-204.

14. Hughes TP, Saglio G, Kantarjian HM, Guilhot F, Niederwieser D, Rosti G, et al. Early molecular response predicts outcomes in patients with chronic myeloid leukemia in chronic phase treated with frontline nilotinib or imatinib. Blood 2014; 123(9): 1353-60.

15. Marin D, Ibrahim AR, Lucas C, Gerrard G, Wang L, Szydlo RM, et al. Assessment of BCR-ABL1 transcript levels at 3 months is the only requirement for predicting outcome for patients with chronic myeloid leukemia treated with tyrosine kinase inhibitors. Clin Oncol 2011; 30(3): 232-38.

16. Branford S, Yeung DT, Parker WT, Roberts ND, Purins L, Braley JA, et al. Prognosis for patients with CML and $>10 \%$ BCR-ABL1 after 3 months of imatinib depends on the rate of BCR-ABL1 decline. Blood 2014; 124(4): 511-18.

17. Vigneri1 P, Stagno F, Stella1 S, Cupri A, Forte S, Massimino $\mathrm{M}$, et al, High BCR-ABL/GUSIS levels at diagnosis of chronic phase CML are associated withunfavorable responses to standard-dose imatinib. Clin Cancer Res 2017; 23(23): 1-10.

18. Irshad S, Butt MA, Joyia A. Frequency of different Bcr-abl Fusion Transcripts in CML Patients in Pakistan. Intl J Agro Veterinary Med Sci 2012; 6(1): 418-23.

19. Marin D, Bazeos A, Mahon FX, Eliasson L, Milojkovic D, Bua M, et al. Adherence is the critical factor for achieving molecular responses in patients with chronic myeloid leukemia who achieve complete cytogenetic responses on imatinib. J Clin Oncol 2010; 28(14): 2381-88

20. Singh O, Chan JY, Lin K, Heng CC, Chowbay B. SLC22A1$\mathrm{ABCB} 1$ haplotype profiles predict imatinib pharmacokinetics in Asian patients with chronic myeloid leukemia. PLoS One 2012; 7(12): e51771.

21. Kumar S, Gupta VK, Bharti A, Meena LP, Gupta V, Shukla J. A study to determine the clinical, hematological, cytogenetic, and molecular profile in CML patient in and around Eastern UP, India. J Family Med Prim Care 2019; 8(7): 2450-55.

22. Bhatti FA, Ahmed S, Ali N. Clinical and hematological features of 335 patients of chronic myelogenous leukemia diagnosed at single centre in Northern Pakistan. Clin Med Insights: Blood Disord 2012; 5(1): 15-24. 